\title{
Construção e validação de estação OSCE para avaliação da habilidade técnica de cricotireoidostomia por punção
}

\author{
Construction and validation of OSCE station to evaluate the technical skill \\ for needle cricothyroidotomy
}

Construcción y validación de la estación de la OSCE para evaluar la habilidad técnica para perforar cricotiroidostomía

Thais Lazaroto Roberto Cordeiro ${ }^{1}$ Juliano Mendes de Souza²

1 Enfermeira. Mestre em Ensino das Ciências da Saúde. Faculdades Pequeno Príncipe. Curitiba, Paraná.

2 Doutor em Clínica Cirúrgica pela Universidade Federal do Paraná. Docente do Programa de Mestrado em Ensino nas Ciências da Saúde das Faculdades Pequeno Príncipe Curitiba, Paraná

\section{RESUMO}

A pandemia ocasionada pela Covid-19, reforçou a necessidade intrínseca de treinamento e avaliação de habilidades técnicas que envolvam a manipulação das vias aéreas, buscando estabilizar a vítima conforme suas demandas. O procedimento de cricotireoidostomia por punção é raro no departamento de emergência, porém é uma habilidade requerida quando as demais alternativas falharam. Nesse sentido, o objetivo deste estudo foi: Construir e validar um cenário simulado na modalidade de Exame Clínico Objetivo Estruturado (OSCE) para avaliação da habilidade técnica de cricotireoidostomia por punção. Estudo metodológico, sendo respeitadas

Autor de Correspondência:

*Thais Lazaroto Roberto Cordeiro. E-mail: thais.lazaroto2014@gmail.com 
as etapas de tradução, construção e validação pertinentes ao método. A pesquisa originou um roteiro para uma estação de OSCE para o ensino da habilidade de cricotireoidostomia por punção. Concluiu-se que a eficácia e confiabilidade da avaliação de habilidades técnicas pode ser realizada dentro de uma OSCE, com instrumentos validados, que forneçam uma avaliação padronizada e replicável em diversos locais.

Palavras-chave: Estudos de Validação. Simulação. Avaliação Educacional. COVID-19.

\begin{abstract}
Covid-19 pandemic reinforced the intrinsic need for training and evaluation of technical skills involving airway manipulation to stabilize the victim according to their needs. Puncture cricothyroidotomy is a rare procedure in the emergency department, although being a required skill when other alternatives fail. In this regard, this study's objective was to build and validate a simulated setting in the Objective Structured Clinical Examination modality to evaluate the technical skill to perform puncture cricothyroidotomy. This is a methodological study, respecting the stages of translation, construction and validation inherent to the method. The research originated a script for the Objective Structured Clinical Examination station for teaching the puncture cricothyroidotomy skills. It was concluded that the evaluation of technical skills can be done with efficacy and reliability in an Objective Structured Clinical Examination setting, using validated instruments that provide a standardized and replicable evaluation in several places.
\end{abstract}

Keywords: Validation Study. Simulation Technique. Educational Measurement. COVID-19.

\title{
RESUMEN
}

La pandemia causada por el Covid-19 reforzó la necesidad intrínseca de capacitación y evaluación de habilidades técnicas que involucran la manipulación de las vías respiratorias, buscando estabilizar a La víctima de acuerdo con sus demandas. El procedimiento de cricotiroidotomía por punción es poco frecuente en el servicio de urgencias, pero es una habilidad requerida cuando otras alternativas han fallado. En este sentido, el objetivo de este estudio fue: Construir y validar un escenario simulado en la modalidad de Examen Clínico Estructurado Objetivo (ECOE) para evaluarla técnica de cricotiroidotomía por punción. Estudio metodológico, siendo respetado como etapas de traducción, construcción y criterios relevantes al método. La investigación resulto en un guion para una estación de la ECOE para enseñar la habilidad de la cricotiroidostomía por punción. Se concluyó que la efectividad y confiabilidad de la evaluación de las competencias técnicas puede realizarse dentro de una ECOE que proporcionan una evaluación normalizada y replicable en varios lugares.

Palabras clave: Estudio de Validación. Simulación. Evaluación Educacional. COVID-19. 


\section{INTRODUÇÃO}

A necessidade de treinamentos que possibilitem a repetição exaustiva de procedimentos visando a aquisição de habilidades motoras são essenciais no departamento de emergência, agravado pelo atual momento pandêmico ocasionado pela Covid-19, que exige dos profissionais destreza técnica principalmente no manejo das vias aéreas objetivando uma boa oxigenação e ventilação, fundamental para a sobrevivência dos doentes acometidos por essa patologia. ${ }^{1,2}$

Quando o médico encontra dificuldades para intubar e oxigenar o paciente, torna-se necessário uma decisão rápida e precisa, pois a falta de oxigenação dos tecidos, em especial, o cerebral levará a danos irreversíveis e risco de óbito iminente, ocasionado pela hipóxia tecidual. ${ }^{3}$

Possibilitar que o profissional vivencie experiências em ambientes seguros e similares ao que encontrará futuramente é uma das possibilidades de metodologia de simulação clínica utilizada para ensino e avaliação de habilidades e competências. No que tange a avaliação utilizando cenários simulados, o Exame Clínico Objetivo Estruturado (OSCE), traduzido de Objective Structured Clinical Examination é utilizado atualmente para provas práticas em acadêmicos na área de saúde, objetivando a avaliação de competências clínicas e procedimentais de forma planejada e estruturada, sendo o ponto principal a objetividade do exame, delineado por objetivo claros e concisos. ${ }^{4}$

Nesse contexto, torna-se essencial a construção de cenários clínicos visando sua replicação nos diversos locais de atuação dos profissionais. Procedimentos técnicos que podem ser necessários no departamento de emergência como a cricotireoidostomia por punção, necessário quando o acesso a via aérea é indicado porém não é possível a intubação orotraqueal ou a utilização de dispositivos supraglóticos. Proporcionar a repetição exaustiva utilizando da simulação familiariza o profissional e aumenta sua segurança e autonomia, construindo uma aprendizagem significativa, após a metodologia ser aplicada para o ensino, podemos construir uma estação visando a avaliação do sujeito daquela habilidade desejada, utilizando da avaliação tipo OSCE. $^{2}$

Partindo desse pressuposto, definimos como o objetivo desse estudo foi: Construir e validar de um cenário de OSCE para a avaliação da habilidade técnica de cricotireoidostomia por punção.

\section{MÉTODO}

Estudo metodológico, seguindo as etapas de tradução, adaptação transcultural, construção e validação de uma estação de OSCE para avaliação de habilidade técnica de cricotireoidostomia por punção.

A pesquisa foi realizada em uma faculdade de medicina da capital do estado do Paraná, escolhida pelo acesso ao Centro de Simulação e uso recorrente de provas na modalidade OSCE em seu currículo acadêmico.

Utilizou-se como base o instrumento disponível na versão atual do Suporte Avançado de Vida no Trauma (ATLS) ${ }^{3}$, da American Heart Association, instituição de relevância internacional em pesquisa e construção de protocolos de emergência, a versão atualmente disponível apenas na língua inglesa indica e preconiza o cumprimento de alguns passos para a eficácia na realização do procedimento. Frente a isso, solicitou-se uma autorização do Colégio Americano de Cirurgiões para a realização da tradução do instrumento e após a aprovação da instituição em questão iniciou-se o processo.

Em seguida, foi elaborado um roteiro para o ensino da cricotireoidostomia por punção utilizando da metodologia de simulação clínica e a partir dele criou-se um instrumento de avaliação da habilidade técnica deste procedimento. 
Foram respeitadas as seguintes etapas: (1) tradução do instrumento para idioma-alvo, (2) síntese das versões traduzidas observando a adaptação transcultural, (3) tradução reversa, (4) validação da síntese por especialistas e (5) estudo-piloto para elaboração do roteiro de simulação, e a partir dele se originou um instrumento de avaliação e formatação para uma estação tipo OSCE, que teve como objetivo: Avaliar a habilidade técnica para a realização da cricotireoidostomia por punção.

$\mathrm{Na}$ tradução do instrumento para idioma-alvo participaram dois especialistas da área de saúde com domínio do idioma inglês e português, conforme especificações do órgão e equivalência de linguística. Posteriormente os autores realizaram a síntese das versões traduzidas e a adaptação transcultural adequando os termos ao âmbito nacional, a tradução reversa seguiu com dois especialistas com domínio dos idiomas em questão para a verificação da fidedignidade ao instrumento original.

Foi realizada a validação do conteúdo por um time de especialistas composto por quatro docentes com vivência em formulação e aplicação de OSCE, além de uma nova avaliação pelos sete experts que auxiliaram na validação do roteiro de simulação. A estação de OSCE também passou por uma validação onde dois profissionais com vivência do procedimento, realizaram o passo a passo, visando a adaptação de tempo e possível viés na observação de um item. Após a compilação das avaliações, realizouse uma nova análise de conteúdo pelos autores para a finalização e teste, originando a versão final do instrumento de avaliação.

Figura 1 - Fluxograma de etapas para tradução, adaptação transcultural, tradução reversa e validação por especialistas roteiro de simulação - Instrumento que originou o roteiro de OSCE.

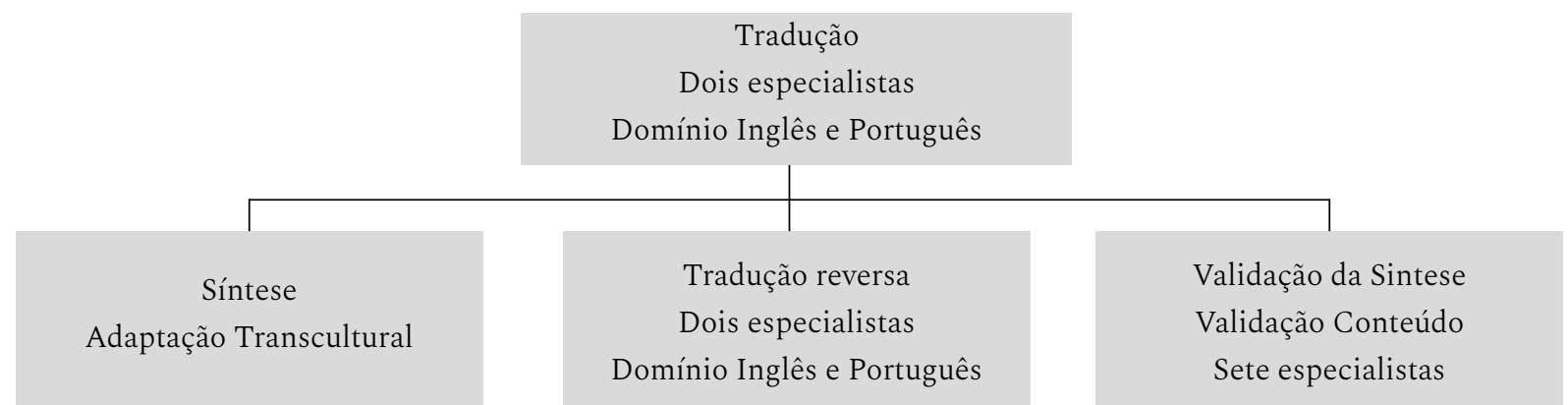

Figura 2 - Fluxograma de etapas para validação do instrumento que originou o roteiro de OSCE.

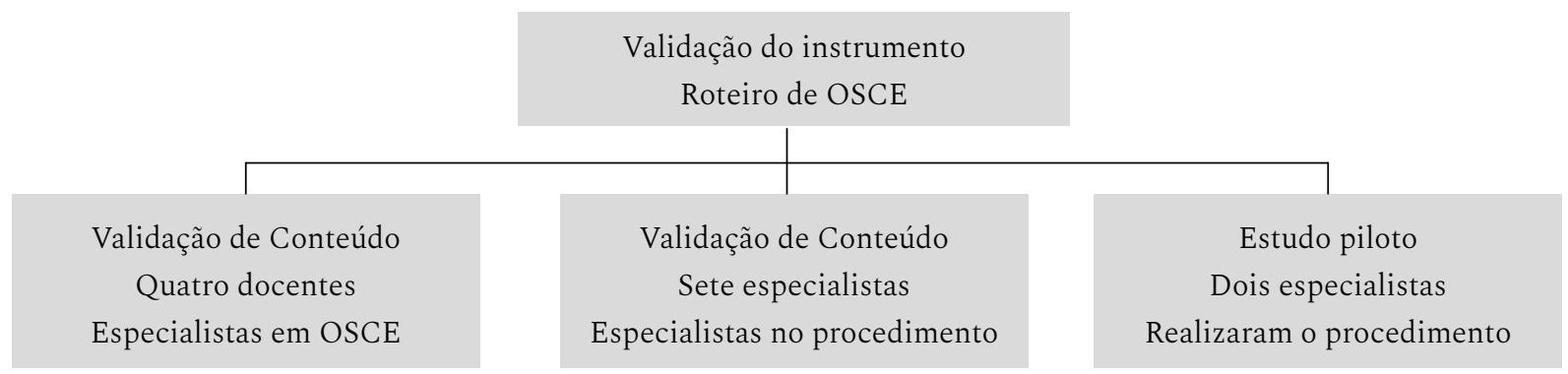


O cenário desenhado buscou avaliar a "Aquisição da habilidade técnica de punção por punção", seguindo a metodologia de simulação clinica com alta fidelidade. Foi utilizado o manequim MegaCode da Laerdal que dentre outros procedimentos permitia a realização de cricotieoidostomia, não havia distratores no cenário e o objetivo era claro ao estudante observando pontualmente a habilidade técnica e não a capacidade de tomar a decisão de indicar o procedimento.

Foi realizado um estudo-piloto com a aplicação do instrumento a um grupo de 11 acadêmicos de medicina que cursavam o primeiro e segundo ano do curso, regularmente matriculados na instituição de ensino escolhida para a pesquisa, que tinham noções de ciências básicas como anatomia e fisiologia. Foram excluídos da pesquisa acadêmicos que já tiveram aula referente ao tema, participaram de liga de trauma ou acompanharam a realização de uma cricotireoidostomia presencialmente.

Após a ciência e a assinatura do Termo de Consentimento Livre e Esclarecido (TCLE), participaram deum workshop com a realização de testes individuais e em grupo, aula expositivo dialogada, prática com simulação, avaliação em formato de OSCE e avaliação de satisfação dos participantes, como facilitador para o conhecimento, aquisição e avaliação da técnica de cricotireoidostomia por punção.

Após a finalização dessas etapas originou-se um instrumento para a avaliação da habilidade de cricotireoidostomia por punção em formato de uma estação OSCE. Essa pesquisa foi aprovada pelo Comitê de Ética da Faculdades Pequeno Príncipe sob o Parecer n 3.164 .915 e respeitou a Resolução CNS 466/2012.

\section{RESULTADOS}

Frente a necessidade de treinamento e avaliação da aquisição da habilidade de cricotireoidostomia por punção, utilizando metodologias de imersão em cenários similares a prática assistencial, buscou-se compreender como se deu a construção de cenários anteriores desses estudos, e principalmente a busca pela fonte de informação que poderia fornecer subsídio para a construção e validação inicialmente de um roteiro de prática simulada, sendo esse o propulsor para a formulação do instrumento avaliativo para ser utilizado em provas práticas tipo OSCE.

Hoje a American Heart Association é a instituição internacional de contribuição mais significativa para o meio científico no atendimento de emergências traumáticas, pediátricas ou cardiológicas, portanto foi a referência utilizada para tradução, validação, adaptação e criação do instrumento.

Cumprindo as etapas metodológicas descritas anteriormente, o estudo gerou um instrumento de avaliação da habilidade, para provas práticas na modalidade tipo OSCE. O instrumento é constituído por 15 itens, onde o acadêmico poderia atingir os seguintes resultados por itens "realizou" (2 pontos), "realizou parcialmente" (1 ponto) ou "não realizou” (0 pontos), o objetivo para atingir a aptidão é o alcance de um score mínimo de 19 pontos conforme descrito no documento (Quadro 1).

O objetivo de aprendizagem foi definido por: "Avaliar a habilidade técnica para a realização da cricotireoidostomia por punção", sendo a competência esperada "Realizar cricotireoidostomia por punção", o instrumento foi subdivido em três etapas, sendo a primeira preparo do material e do paciente, seguido pela realização do procedimento propriamente dita e a finalização.

Alguns itens recebem destaque no instrumento por serem considerados obrigatórios, ou seja, caso não fossem realizados o aluno automaticamente é considerado inapto para o procedimento.

$\mathrm{Na}$ validação do instrumento certificou-se que o instrumento considerou todos os aspectos 
relevantes, ordem de realização e passos definidos como essenciais para a avaliação, em modo que cada item tivesse clareza suficiente para o avaliador e que fossem passíveis de observação.

Quadro 1 - Instrumento de avaliação, modalidade OSCE para avaliação da habilidade técnica de cricotireoidostomia por punção.

\begin{tabular}{|c|c|c|c|c|}
\hline \multicolumn{5}{|c|}{ Cricotireoidostomia por Punção } \\
\hline \multicolumn{2}{|r|}{ Primeira Etapa: Preparo do material e do Paciente } & Realizou & $\begin{array}{l}\text { Realizou par- } \\
\text { cialmente }\end{array}$ & Não finalizou \\
\hline 1 & $\begin{array}{l}\text { Retirar conector da cânula orotraqueal } n^{\circ} 3,0 \text {, para a } \\
\text { adaptação do cateter sobre agulha }\end{array}$ & & & \\
\hline 2 & Posicionar o paciente em decúbito dorsal & & & \\
\hline 3 & Aspirar 2ml de Solução Fisiológica & & & \\
\hline 4 & $\begin{array}{l}\text { Acoplar um cateter sobre agulha } \mathrm{n}^{\circ} 14 \text { a essa seringa de } \\
5 \mathrm{ml}\end{array}$ & & & \\
\hline 5 & $\begin{array}{l}\text { Solicite que um auxiliar posicione o pescoço levemente } \\
\text { estendido }\end{array}$ & & & \\
\hline 6 & Realizar antissepsia do pescoço & & & \\
\hline 7 & Palpar a membrana cricotireóidea & & & \\
\hline \multicolumn{5}{|c|}{ Segunda Etapa: Realização do Procedimento } \\
\hline 8 & $\begin{array}{l}\text { Estabilizar a traqueia com o polegar e o indicador da } \\
\text { mão não dominante }\end{array}$ & & & \\
\hline 9 & $\begin{array}{l}\text { Perfurar a pele na linha média com o cateter conectada } \\
\text { na seringa, diretamente sobre a membrana cricotireóidea }\end{array}$ & & & \\
\hline 10 & Direcionar o cateter em ângulo de $45^{\circ}$ para baixo & & & \\
\hline 11 & $\begin{array}{l}\text { Aplicar pressão negativa na seringa e observar a as- } \\
\text { piração de ar, significando a entrada na traqueia }\end{array}$ & & & \\
\hline 12 & Retire a agulha do cateter & & & \\
\hline \multicolumn{5}{|c|}{ Terceira Etapa: Finalização } \\
\hline 13 & Fixar o cateter no pescoço do doente & & & \\
\hline 14 & Observe a expansibilidade dos pulmões & & & \\
\hline 15 & $\begin{array}{l}\text { Realizar ausculta do tórax para verificar se a ventilação } \\
\text { está adequada }\end{array}$ & & & \\
\hline
\end{tabular}




\section{Resultado:}

( ) APTO para realizar a cricotireoidostomia por punção;

( ) INAPTO para realizar a cricotireoidostomia por punção;

( ) APTO para realizar a cricotireoidostomia por punção

( ) INAPTO para realizar a cricotireoidostomia por punção

Será atribuído 2 (dois) pontos por item para o acadêmico que realizou, 1 (um) ponto caso tenha realizado parcialmente e 0 (zero) pontos caso não tenha realizado o item. Os Itens em amarelo são pontos de corte, caso não sejam realizados o discente estará inapto para completar o procedimento.

A pontuação máxima será de 30 pontos e a mínima de 0 pontos. Para ser apto a realização do procedimento o discente deve atingir a pontuação mínima de 19 pontos.

Utilizado com referência, passo a passo para a realização de cricotireoidostomia por punção, proposto pelo ATLS $10^{\circ}$ edição, 2018.

\section{DISCUSSÃO}

A simulação clínica é uma metodologia que permite o ensino e avaliação de procedimentos com cenários similares aos da prática assistencial. Referente a avaliação do OSCE, tem sido utilizado com frequência inclusive para provas de Revalidação de diplomas em contexto nacional. Desenvolvido na década de 70 na Escócia, por Harden e Gleeson $(1979)^{4}$, objetiva a avaliação de competências clínicas em acadêmicos de medicina. Desde então o método só tem crescido e disseminado nas universidades pelo seu reconhecimento internacional, sendo um instrumento válido, fidedigno e efetivo. 5,6

Segundo Harden e Gleeson ${ }^{4}$, o OSCE é definido como:"Uma abordagem para a avaliação da competência clínica em que os componentes de competência são avaliados de forma planejada e estruturada com atenção para a objetividade do exame” Para a eficácia do OSCE é necessário identificar as competências que quer avaliar, sendo necessário um planejamento rigoroso.,
Quando nos deparamos com um objetivo de aprendizagem voltado para aquisição de uma habilidade, pode se utilizar a nomenclatura OSPE, que constitui uma vertente do OSCE que tem por objetivo a avaliação voltada para procedimentos e não habilidades clínicas. A avaliação consiste em estações onde os acadêmicos realizam as tarefas solicitadas sendo assistidos por avaliadores, durante um período de tempo, com o intuito de atingir objetivos de aprendizagem delimitados previamente, como por exemplo a execução de procedimentos, exame físico ou anamnese. As estações devem durar em torno de 5 minutos. ${ }^{4,5}$

A construção de instrumentos fidedignos, confiáveis e reprodutíveis em qualquer situação é necessária para a organização do processo de aplicação do OSCE. Para a realização da avaliação de desempenho do estudante tornou-se necessário a construção de um instrumento que realmente realizasse a medida correta do objetivo de aprendizagem esperado, nesse caso a avaliação para a aquisição de habilidade de 
cricotireoidostomia por punção. ${ }^{8}$ Revisão sistemática realizada em 2018, discorre sobre a importância da validação de instrumentos para a aplicação de OSCE, referindo essa etapa como primordial na confiabilidade da avaliação, além disso, facilitando sua aplicação pelos docentes que poderão utilizarse desses recursos já validados para avaliação dos discentes nessa habilidade. ${ }^{9}$ Estudo realizado em Florianópolis, discorre sobre a aplicação de OSCE para acadêmicos do curso de farmácia, o relato de experiência relata sobre as etapas utilizadas para a construção de simulação para o ensino de habilidades aos estudantes, a aplicação da OSCE favorece o equilíbrio psicoemocional e ambiental inserindo o acadêmico em situações similares à prática assistencial, favorecendo seu treinamento, além de oferecer uma imersão em pressões que podem ocorrer durante a vida profissional dos participantes. ${ }^{10}$ Miller $^{11}$ durante uma conferência corrobora com a necessidade de planejamento e sustentação do uso método através de estratégias e construção de instrumentos fidedignos que propõem a medir exatamente o objetivo de aprendizagem delimitado previamente, em um processo avaliativo isso se torna essencial pela sua aplicabilidade ser justa a todos os atores participantes, afinal ela produzirá uma nota/conceito ao fim com demasiada importância acadêmica.

A eficácia da metodologia de simulação clínica para avaliação está alinhada à construção e validação de roteiros os quais conduzam a prática de forma padronizada, respeitando todas as etapas do método. Propor desenhos educacionais que ofereçam interação e imersão dos acadêmicos da área da saúde utilizando diversas metodologias de aprendizagem e avaliação podem favorecer o crescimento de autonomia e segurança do profissional em formação, visando como produto final o aumento da segurança do paciente. Sendo necessários mais estudos para a produção e validação de instrumentos fidedignos na avaliação de competências de acadêmicos na área da saúde.

\section{CONCLUSÕES}

Concluiu-se que o instrumento de avaliação prática na modalidade OSCE com o objetivo de aprendizagem da habilidade técnica para cricotireoidostomia por punção está validado no conteúdo e pode ser utilizado como uma padronização para aplicação em diversos cenários acadêmicos e assistenciais.

\section{REFERENCIAS}

1. Conselho Federal de Enfermagem (BR). Resolução $n^{\circ}$ 641/2020, dispõe sobre a utilização de Dispositivos Extraglóticos (DEG) e outros procedimentos para acesso à via aérea, por Enfermeiros, nas situações de urgência e emergência, nos ambientes intra e pré-hospitalar [Internet]. Brasília; 2020. [cited 2021 Jun 2].Available from: http://www. cofen.gov.br/resolucao-cofen-no-641-2020_80392.html

2. Nimbalkar A, Patel D, Kungwani A, Phatak A, Vasa R, Nimbalkar S. Randomized control Trial of high fidelity VS low fidelity simulation for training undergraduate students in neonatal resuscitation. BMC Res Notes. dezembro de 2015;8(1):636.

3. American College of Surgeons. Advanced Trauma Life Suport. 10. ed. Chicago: Copyright; 2018.

4. Harden RM, Stevenson M, Downie WW, Wilson GM. Assessment of clinical competence using objective structured examination. Br Med J, 1975, 1(5955), 447-451.

5. Neves RS, Barros AF, Esper MM de A, Bezerra TJN. Avaliação do exame clínico objetivo estruturado (OSCE) por estudantes e docentes de graduação em enfermagem. Com. Ciências Saúde 27(04):309-16.

6. Duarte AS, Silva AP, Soares AS, Roque ABB, Jesus Neto JF, Sefer CCI. Avaliação do desempenho de estudantes de medicina no OSCE: o papel do estudo prévio. Acervo Saúde12(10):e4506.

7. Neves FF, Pazin Filho A. Construindo cenários de simulação: pérolas e armadilhas. Sci Med. 2018;28(1):ID28579.

8. Jeffries PR. A framework for designing, implementing, 
and evaluating: Simulations used as teaching strategies in nursing. Nursing education perspectives. 2005,26(2), 96103.

9. Rosso LH. Elementos estruturais do Exame Clínico Objetivo Estruturado (OSCE) no ensino em enfermagem para sua operacionalização: revisão sistemática. [dissertação de mestrado]. Porto Alegre: Universidade Federal do Rio Grande do Sul; 2018.

10. Galato D, Alano GM, França TF, Vieira AC. Exame clínico objetivo estruturado (ECOE): uma experiência de ensino por meio de simulação do atendimento farmacêutico. Interface - Comunicação, Saúde, Educação;15(36):309-20.

11. Miller, J. et al. Strategies Associated With OSCE Simulation, Anxiety, and Clinical Competency in a Family Nurse Practitioner Program. Nursing Education Research Conference. Washington, 2018. 